\title{
Análisis de las tendencias en contenidos y plataformas de comunicación de marca en España
}

\section{Analysis of content trends and brand communication platforms in Spain}
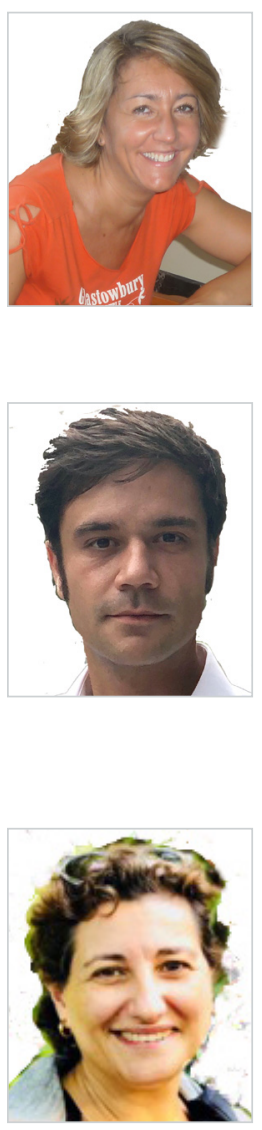

Ana Sebastián Morillas. Doctora en Comunicación Audiovisual y Publicidad por la Universidad de Valladolid. Actualmente es profesora contratada doctora e imparte docencia en programas de grado y posgrado. Sus principales líneas de investigación están relacionadas con la planificación estratégica de la marca, la publicidad, la comunicación y el marketing. Ha sido miembro del RCC en la Universidad de Harvard (Cambridge, Massachusetts) y ha formado parte del Comité Científico de varios Congresos de Publicidad, participando como ponente en diferentes Congresos Nacionales e Internacionales. Miembro de la Asociación Española de Investigación en Comunicación, y de la Asociación Icono 14. También forma parte del Grupo de Investigación Reconocido (GIR): "Gestión de marca y procesos de comunicación" en la Universidad Complutense.

Universidad de Valladolid, Segovia, España

anaseb@hmca.uva

ORCID: 0000-0001-9073-3483

Abel Monfort. Doctor, SFHEA. Director del Departamento de Negocios para Estudios de grado en ESIC Business \& Marketing School. Profesor de doctorado acreditado por ANECA y miembro principal de la Academia de Educación Superior. Fue profesor académico visitante en el Centro Hoffman para la Ética Empresarial (Bentley University). Su investigación se centra en la comunicación de la RSE en las redes sociales. Monfort es investigador asociado en el Laboratorio de Investigación de ESIC Business \& Marketing School y miembro de la Cátedra de Ética Económica y Empresarial en la Universidad Pontificia de Comillas. También es miembro del grupo de investigación "Gestión de marca y procesos de comunicación" que posee la Escuela de Comunicación de la Escuela de la Universidad Complutense.

ESIC Business \& Marketing School, Madrid, España

abel.monfort@esic.edu

ORCID: 0000-0002-3713-7102

Belén López. Doctora en Filosofía. Profesora en ESIC, Business and Marketing School, Madrid, reconocida por la acreditación ANECA en 2013. Es investigadora asociada de la Unidad de Investigación Permanente (UPI) en ESIC desde 2017. Su investigación se centra en la RSE y comunicación. Además, es miembro del grupo de investigación "Gestión de marca y procesos de comunicación" de la Universidad Complutense, desde 2011. Miembro de la Academia de Negocios Internacionales y de la Academia de Administración desde 2010. Ha participado en varios proyectos internacionales, así como en conferencias y eventos de este tipo en los últimos años.

ESIC Business \& Marketing School, Madrid, España

belen.lopez@esic.edu

ORCID: 0000-0001-9010-6068

Cómo citar este artículo: Sebastián Morillas, A.; Monfort, A.; López, B. (2018). Análisis de las tendencias en contenidos y plataformas de comunicación de marca en España. Doxa Comunicación, 27, pp. 147-171. 
Recibido: 31/08/2018 - Aceptado: 19/10/2018

\section{Resumen:}

Esta investigación define las tendencias de las plataformas de contenido y comunicación utilizadas por marcas corporativas y comerciales en España, así como la gestión de relaciones entre marcas y consumidores. Se ha utilizado el método Delphi con cuatro paneles compuestos por 15 expertos en diferentes temáticas relacionadas con el objeto de estudio. Los resultados muestran que las marcas corporativas colocan la identidad como un motor del contenido que generan, y afirman que la responsabilidad social corporativa, la reputación y la cultura corporativa son áreas capaces de generar contenido relevante. Destaca la co-creación, el diálogo con las partes interesadas y el contacto con los líderes de opinión, para crear experiencias de marca y ser reconocidos.

Palabras clave:

Estrategias de comunicación, marca comercial, marca corporativa, Delphi, Stakeholders.
Received: 31/08/2018 - Accepted: 19/10/2018

\section{Abstract:}

This research delves into the trends in content and communication platforms used by corporate and commercial brands in Spain, and also defines the management of current relations between brands and their consumers. This research has been carried out using the Delphi method, designing four panels made up of 15 experts in different topics related to the object of study. Results show that corporate brands place identity as an engine of the content they generate, and also claim that corporate social responsibility, reputation and corporate culture are areas capable of generating relevant content. Finally, the article emphasizes co-creation, dialogue with stakeholders and contact with opinion leaders, in order to create brand experiences and be recognized.

\section{Keywords:}

Communication strategies, commercial brand, corporate brand, Delphi, stakeholders.

\section{Introducción: Marco teórico}

\subsection{La estrategia de comunicación en la marca corporativa}

En este epígrafe se va a desarrollar el concepto de marca corporativa, las implicaciones de su gestión en el resto de intangibles, la forma en que la arquitectura de marcas incide en la comunicación con stakeholders y el efecto de la teoría de los grupos de interés. Este conjunto de vinculaciones permitirá una correcta aproximación a los objetivos del estudio y justificar la relación con la marca comercial.

La marca corporativa añade valor a productos y servicios ofertados por la empresa (Ind, 1997). Por lo tanto, influye en la forma en que se desarrollan las marcas comerciales. La influencia dependerá de la eficacia en la gestión de los intangibles empresariales, de la arquitectura de marcas -forma en que se relacionan entre sí las distintas marcas de la empresa- (Aaker, 2004), y de las relaciones con los grupos de interés. Como paso previo a la explicación de estos factores, hay que identificar el significado de marca corporativa.

En primer lugar, se puede afirmar que la marca corporativa representa la identidad de la empresa: valores, visión y cultura corporativa (Balmer \& Gray, 2003; Hatch \& Schultz, 2010). Es decir, la marca corporativa expresa identidad verbal y visual pero, esencialmente, comportamientos (Knox \& Bickerton, 2003). Dicho de otro modo, la gestión de la marca corporativa transmite lo que una empresa es y espera llegar a ser (Schultz \& de Chernatony, 2002). Un buen desempeño de las variables de identidad de marca corporativa favorecerá la diferenciación, los costes y la transparencia (Hulberg, 2006). Por lo tanto, definirá la futura comunicación, las plataformas a utilizar y la búsqueda de tendencias desde los departamentos correspondientes. Al tener una visión global y transversal, la identidad de la marca corporativa será transmitida en menor 
o mayor grado a las marcas comerciales de la empresa, que en última instancia son las que difundirán el mensaje a los consumidores y a otros grupos de interés.

En segundo lugar, se desarrollan las implicaciones de la marca corporativa en el resto de intangibles. Según se desprende de la revisión que se describe en los guiones siguientes, la relación entre comportamientos (identidad) y comunicación corporativa recibe apoyo de la gestión de los llamados intangibles empresariales. Los departamentos de Responsabilidad Social Corporativa (RSC), reputación o cultura corporativa generan contenido relevante para transmitir a los diferentes públicos y crean promesas de marca. Consecuentemente, la gestión de intangibles conlleva integración, complementariedad y construcción de marca. A continuación, se explican las principales áreas: la reputación corporativa es “ $a$ set of collective evaluations that is evoked in different audiences by the behaviour of a company and predisposes the audience towards support or resistance" (Carreras et al., 2013: 86) y conlleva la medición para el posterior desarrollo de la identidad de marca y su comunicación. Esto ayudará a establecer las políticas de comunicación corporativa más adecuadas y a gestionar corectamente el comportamiento de los diferentes departamentos de intangibles.

La responsabilidad social corporativa (RSC) es el compromiso empresarial por la actuación sostenible, que las compañías integran en su estrategia corporativa, que tiene en cuenta a los stakeholders y busca la creación de valor económico y social (Lindgreen et al., 2012; Windsor, 2006; López \& Fornes, 2015; López \&Villagra, 2017); La marca desempeñará un papel destacable y, si la que la empresa quiere ser socialmente responsable, ésta debe alinear identidad y compromiso en RSC (Villagra \& López, 2013). Es decir, desarrollará acciones sostenibles vinculadas con la marca corporativa. Estos comportamientos podrán ser trasladados a los públicos mediante el uso de herramientas comunicativas. Las empresas podrán poner el foco de la acciones de RSC en la marca corporativa o en la comercial. Sin embargo, dada la necesidad de transversalidad en la ética de la empresa, es más recomendable vincular los valores de RSC con la marca corporativa, puesto que la RSC es un departamento global que tiene un entendimiento completo de la visión y misión corporativa (Monfort \& Villagra, 2016).

La cultura organizacional se relaciona con el patrón de comportamiento que un grupo de individuos (empleados) ha investigado, descubierto o desarrollado en el proceso de aprendizaje para afrontar la integración y la adaptación (Schein, 1984: 3) dentro y fuera de la empresa. Será ventaja competitiva si desarrolla matices internos que sean valiosos, diferentes y difícilmente imitables por otras compañías (Barney, 1986). Las empresas son más atractivas para trabajar cuando son percibidas como respetables, con buena imagen de producto o como un espacio que fomenta el buen clima laboral (Highhouse et al., 1999), así como la buena percepción del sector de actividad, los beneficios obtenidos o la familiaridad con la empresa (Cable \& Graham, 2000). En el desarrollo de la cultura corporativa se tienen en cuenta la identidad de la marca y la forma en que es comunicada a sus miembros (Hatch \& Schultz, 2003). En resumen, la cultura organizacional favorece un comportamiento en los empleados capaz de ser comunicado a los stakeholders (Galbreath, 2010) y, por lo tanto, mejora el posicionamiento de la marca. El comportamiento esperado en los empleados tendrá mucha relación con lo que la empresa dice ser a través de su marca corporativa.

Consecuentemente, los intangibles y la marca presentan un conjunto de influencias bidireccional. La reputación, responsabilidad social y cultura corporativa repercuten en la gestión de marca y en la forma en que ésta se posicione con la comunicación. Del mismo modo, la identidad de la marca actuará de guía para el desarrollo de cada una de las actuaciones 
que desarrolla el departamento de reputación (los atributos que se medirán estarán relacionados con la identidad), el de RSC (la identidad definirá los valores responsables) y en el de cultura corporativa (lo que se comparte como miembro de una organización descansa en la identidad de la marca corporativa). Por esa razón, esta investigación tendrá en cuenta la influencia de todo este conjunto de intangibles en la comunicación que llevan a cabo las marcas corporativas en España en la actualidad y con visión de futuro.

En tercer lugar, la comunicación de marca corporativa estará muy delimitada por la arquitectura de marcas de la empresa. Este concepto se refiere a la forma en que se organiza el portfolio de marcas de la empresa y las relaciones que se establecen entre las diferentes marcas (Aaker, 2004) y la marca corporativa. Tal y como se ha dicho, la marca corporativa influirá en la comunicación de las marcas comerciales. Será la arquitectura de marca la que delimite el nivel de influencia de la identidad corporativa en la comunicación de los productos y servicios.

En líneas generales, la arquitectura de marcas se podrá dividir en las siguientes estrategias: 1) branded house, una misma denominación (marca) para todos los servicios y productos de la organización (Kotler \& Keller, 2006), 2) endorsed brands, cuando una marca institucional respalda con su identidad corporativa al conjunto de marcas de la organización (Aaker \& Joachimsthaler, 2000a) y 3) house of brands, estrategia en la que la empresa presenta cada producto o marca comercial con una denominación (marca) e identidad visual diferente (Aaker \& Joachimsthaler, 2000b). La elección de cada una de ellas limitará o potenciará la comunicación y relación que tiene la empresa con los stakeholders, puesto que el foco comunicacional descansará en la marca corporativa o comercial dependiendo de la estrategia. Tanto en la arquitectura de branded house como en endorsed brand, la marca corporativa influirá de manera relevante en el estilo comunicativo de las marcas comerciales, puesto que la identidad corporativa dará lugar a la esencia de los productos y servicios comunicados.

En cualquiera de los casos, el mensaje de la marca corporativa debe ser coherente con la identidad y debe evitar que los contenidos generados acaben "centrándose cada uno en su parcela" (Capriotti, 2007: 53). Por lo tanto, la comunicación debe reforzar una imagen determinada a partir de la identidad. La literatura expone herramientas y plataformas de comunicación de marca corporativa, tales como:

- Publicidad corporativa (en prensa, radio, televisión, cine, exteriores -anuncios en prensa, cuñas, spots, vallas y monopostes, etc.)- (Ortega Martínez, 1997: 78) o Internet (formatos integrados, flotantes, redes sociales, e-mail marketing o website, etc.) (Pintado et al., 2010).

- Relaciones Públicas, tales como revista interna, tablón de anuncios, carteles, eventos, memoria anual, folletos, visitas guiadas, publicity, congresos, jornadas, concursos, patrocinio, inauguraciones, colaboración social o programas de acción social (Reinares \& Calvo, 2001: 25).

Todas estas herramientas y plataformas tendrán como objetivo comunicar las acciones de la marca corporativa, teniendo como premisa la creación de una imagen acorde con la identidad corporativa. Los intangibles serán capaces de generar contenido comunicable a través de estos medios. En arquitecturas de marca branded house o endorsed brand, la comunicación de marca corporativa reforzará la percepción de los productos y servicios entre los consumidores. Sin embargo, se observa que las herramientas de comunicación descritas no sólo están organizadas para impactar en consumidores, sino que la marca presenta una visión multistakeholder. Es decir, para la marca corporativa existen otros públicos y será la gestión de las relaciones con los stakeholders la que determine el qué y a quién se comunica desde el área de comunicación cor- 
porativa. Consecuentemente, en cuarto lugar, conviene mencionar que las estrategias de comunicación estarán limitadas por la relación que adquiera la marca institucional con el conjunto de los denominados stakeholders. La teoría ha definido a los stakeholders como aquellos grupos o individuos que puedan afectar o ser afectados por el logro de los propósitos de una organización (Freeman, 1984). En líneas generales, los stakeholders tendrán intereses o expectativas con relación a la empresa. Entre los que la literatura ha destacado, se pueden citar: los intereses materiales, políticos, de pertenencia, acceso a la información e intereses simbólicos o espirituales (Wartick \& Wood, 1998).

La organización, y por lo tanto la marca corporativa, deberá hacer un ejercicio de jerarquización que permita detectar a los grupos de interés (accionistas, empleados, consumidores, comunidad, proveedores, etc.) y gestionar adecuadamente la relación. Es decir, necesita conocer quiénes son los stakeholders, qué esperan, en qué fundamentan sus expectativas y cómo se entiende su relación con la empresa (Fernández \& Bajo, 2012).

Una forma tradicional de establecer esta jerarquización ha sido a través del análisis de los siguientes atributos: su capacidad de influencia en la compañía, la legitimidad para la relación empresa-stakeholder; y 3) la urgencia que tiene su pretensión (Mitchell, Agle \& Wood, 1997). Tal y como se ha dicho, esta investigación procura conocer cómo observan el proceso de relación desde la gestión de marcas en España. En todo el proceso de gestión de grupos de interés cobra especial relevancia la necesidad del diálogo. Este diálogo sirve para conocer lo que se espera de la empresa y para fortalecer la identidad y correcto desempeño de la organización para incrementar su transparencia (Colleoni, E., 2013). En este sentido, al hablar de social media, las organizaciones muestras su responsabilidad con el diálogo cuando son accesibles vía redes sociales (Kollat \& Farache, 2017).

Mediante la promoción del diálogo, las empresas puedan incrementar su engagement. Así, Hurst y Ihlen (2018) proponen un proceso para el incremento de la interacción con los públicos: 1) asegurar el compromiso interno (alta dirección y empleados), 2) tener en consideración las expectativas de los grupos de interés para hacer un mapa de actividades responsables, 3) "cerrar el círculo" con la comunicación a los stakeholders de que su participación ha sido parte del proceso de construcción y 4) comunicar los resultados de las acciones.

No obstante, estudios actuales (Illia et al., 2015) han demostrado que, pese a desarrollar espacios adecuados para el diálogo, las empresas dialogan y conversan sobre temas que interesan únicamente a la organización y no favorecen un diálogo abierto en el que los grupos de interés puedan expresar claramente sus posturas y expectativas. Además, algunos autores muestran que las compañías apenas utilizan sus redes sociales para comunicar sus acciones de responsabiliad social y para generar engagement con sus stakeholders (Chomvilailuk \& Butcher, 2018).

En definitiva, la comunicación de la marca corporativa también estará delimitada por la jerarquización de sus grupos de interés. Así, las empresas con una arquitectura branded house (una misma marca para todos los productos y servicios) tendrán un mapa de públicos muy amplio, puesto que abarcarán a todos los grupos y las acciones de sus intangibles influirán notablemente en todos ellos. Por otro lado, las empresas con una estrategia de branded house (donde marca corporativa respalda a marcas comerciales) pueden dedicar la comunicación a unos públicos concretos (accionistas, empleados, proveedores...) y reforzar el impacto a los consumidores mediante la comunicación de sus marcas comerciales. En los porta- 
folios basados en house of brands (multimarcas), la marca corporativa comunicará a stakeholders muy concretos, mientras que las marcas comerciales se relacionarán exclusivamente con los consumidores finales.

Este punto ha permitido conocer la dificultad de gestión comunicacional de la marca corporativa. Con esta investigación se podrá conocer cuáles son las tendencias de comunicación desde la óptica empresarial y el estado actual de la gestión de relaciones con los grupos de interés. Previamente, es fundamental revisar la literatura en torno a la gestión de comunicación en marcas comerciales. Como se ha indicado en este epígrafe, la relación entre marcas corporativas y comerciales es muy estrecha, puesto que la arquitectura de marcas y la estrategia de relación con los grupos de interés provocan que la identidad de la marca matriz y la labor de sus intangibles se traslade a las marcas comerciales de una manera u otra.

\subsection{La estrategia de comunicación en la marca comercial}

La marca comercial representa un importante recurso para la empresa porque genera valor promocional a la marca (Cohen, 1986). Por lo que la publicidad y el marketing son herramientas fundamentales para dar a conocer los productos y servicios de la marca. Es por ello que Day y Wensley (1983) argumentan que los proveedores pueden contribuir a una orientación estratégica de la empresa, a través de la formulación y aplicación de la estrategia a nivel corporativo. Esto nos hace darnos cuenta de que marca comercial y corporativa van unidas. En este apartado se va a desarrollar el concepto de marca comercial, y de cómo la comunicación estratégica y la aplicación de estrategias de comunicación son fundamentales para llegar al público objetivo, y para conectar la marca con el consumidor. Por lo que esta vinculación permitirá la aproximación a los objetivos de la investigación.

La principal forma que tiene la marca comercial de darse a conocer entre sus públicos, es a través de la comunicación, en concreto a través de estrategias de comunicación y de marketing. La comunicación es la base de todas las organizaciones y sus actividades. Desde hace mucho tiempo los investigadores han hecho hincapié en la estrecha relación entre comunicación y organizaciones. Barnard (1938/1968) explicó que la comunicación era fundamental para una empresa. Pero fue Weick (1979) quien comenzó a interesarse por la importancia de la comunicación en las organizaciones, argumentando que las empresas se componen de una variedad de relaciones interpersonales formales e informales entre las personas, que deben mantenerse constantemente. Asimismo Tuñez (2012) hace hincapié en la importancia de que las organizaciones son el resultado de su comunicación con el entorno y consigo mismas. Es necesario comprender, planificar y desarrollar una gestión comunicativa eficaz.

Las empresas se han dado cuenta del valor de la estrategia de comunicación y de cómo a través de la estrategia se pueden transmitir valores, emociones y sensaciones, que lleven a la acción. En el caso de una compañía, la estrategia sería el mejor camino para cumplir el objetivo empresarial que se debe lograr (Sebastián et al., 2012).

Por eso, toda organización que quiera comunicar de manera eficaz sus mensajes y cumplir con los objetivos marcados, debería diseñar una estrategia de comunicación. Los pasos a seguir consistirían en: realizar un análisis de la situación actual, marcar los objetivos organizacionales y de comunicación, identificar los públicos objetivos, dar coherencia a los mensajes, seleccionar los medios de comunicación adecuados y determinar los criterios para medir y evaluar los resultados (Alberto Pérez, 2008; Alard \& Monfort, 2017). 
Todas las empresas deberían diseñar estrategias de comunicación para lograr sus objetivos, aunque los pasos pueden variar en función de la empresa que diseñe la estrategia, pero todas deben fijar los objetivos antes de establecer la estrategia. Las empresas necesitan saber de dónde parten, cómo son, que están haciendo bien o mal, hacia dónde van. Todo ello forma parte del comportamiento estratégico de las organizaciones. Igor Ansoff (1965), ya propuso una matriz muy popular de cuatro estrategias: penetración en el mercado, desarrollo del producto, desarrollo del mercado y diversificación. Años más tarde, Porter (2002) definió tres estrategias genéricas para que las empresas alcanzaran una ventaja competitiva: la estrategia de diferenciación, la estrategia de liderazgo de costos y la estrategia de enfoque o segmentación. La mayoría de las empresas que están en el mercado, utilizan muchas de estas estrategias para conseguir una diferenciación entre su marca y la de la competencia.

No hay que confundir la comunicación estratégica con la estrategia de comunicación, porque no son equivalentes, aunque las marcas comerciales utilizan ambas para llegar a sus públicos. La comunicación estratégica es un campo multidisciplinar de conocimiento definido como comunicación con propósito, que una organización utiliza para planificar, ejecutar y cumplir su misión general en relación con los diferentes grupos de interés internos y externos (Hallaban et al., 2007). Es decir, la comunicación estratégica es una parte dentro de la estrategia de comunicación, así lo corrobora Falkheimer (2014), es un elemento más, pero necesario, para el desarrollo de la organización. Sin embargo, la estrategia de comunicación implica una investigación y normalmente se diseña para resolver los posibles problemas que pueda tener la marca comercial. Va a permitir ver las cosas a medida que van cambiando, es una guía imprescindible para las empresas. Es importante resaltar algunos conceptos claves de la estrategia de comunicación que aportan soluciones comunicacionales y garantizan la eficacia de la campaña: público objetivo al que nos dirigimos, qué problema queremos resolver, posicionamiento de nuestro producto, etc., (García, 2001).

Todos estos elementos crean la diferencia entre buenas y malas, eficaces e ineficaces, arriesgadas y tradicionales estrategias de comunicación. Las empresas deben pensar en una estrategia comercial que ayude a alcanzar su ventaja competitiva en el mercado, y para conseguirlo necesitan tener un mayor conocimiento del consumidor, inteligencia competitiva y análisis de tendencias (Osler, 2003). Por eso, "en un entorno de negocios donde la mayoría de las cosas son similares, la compañía que disponga de una buena estrategia de comunicación tendrá una ventaja determinante" (Sebastián et al., 2012).

Es importante señalar que las marcas comerciales necesitan de las estrategias de comunicación para obtener una ventaja competitiva en el mercado y para poder reflejar el insight que conecte la marca con el consumidor. Está vinculación hará que la marca comercial forme parte de la vida de las personas. Hoy en día, las redes sociales y las nuevas tendencias en comunicación digital permiten a las empresas establecer conversaciones con las audiencias. Especialmente, en aquellas industrias que tienen una aproximación más natural a los medios sociales, como el sector del entretenimiento (Arroyo, Martín \& Farfán, 2013).

En conclusión, la revisión de la literatura ha permitido observar que, más allá de aspectos visuales, la marca corporativa es entendida como el propósito y aspiraciones de la empresa que genera valor a toda la organización, mediante la definición de unos comportamientos que quedan recogidos en su identidad corporativa. La relación entre marca corporativa 
y comercial podrá ser más o menos pronunciada en función de la arquitectura de marcas de cada compañía, ya que ésta delimita las relaciones de la empresa con el conjunto de sus marcas.

Cuando la identidad de la marca corporativa y la gestión de su portafolio estén ambos bien definidos, las acciones de los intangibles y las relaciones con los stakeholders serán los que delimiten la creación de mensajes específicos que serán desarrollados por herramientas y plataformas de comunicación, destinadas a incrementar la notoriedad y reputación de la empresa. Por otro lado, aunque la comunicación de marca corporativa puede suponer un apoyo a la labor de marcas comerciales en arquitecturas branded house y endorsed brand, la marca comercial sigue siendo la promesa fundamental para un público concreto como son los consumidores. Por lo tanto, parece necesario conocer cuáles son los pasos a seguir para diseñar eficaces estrategias de comunicación, y cuáles son las estrategias más utilizadas por las marcas descritas en el marco teórico.

La literatura de comunicación de marca corporativa y comercial viene definida por las herramientas tradicionales (publicidad, marketing y relaciones públicas). Consecuentemente, parece necesaria una actualización y delimitación de las estrategias situadas a la vanguardia. Del mismo modo, la comunicación de marcas comerciales ha incidido en desarrollar buenas estrategias de comunicación, para diferenciarse en el mercado y alcanzar una ventaja competitiva. Por eso las marcas utilizan estrategias de diferenciación, de liderazgo de costos, de segmentación, etc., para llegar al público objetivo y lograr conectar con él. Las empresas utilizan la comunicación integrada dentro de las estrategias de comunicación, para lograr la eficacia en sus campañas publicitarias, y así asociar la marca al consumidor.

Por esta razón es pertinente conocer cuáles son las tendencias en cada una de ellas y qué están haciendo ahora las empresas en sus relaciones con los públicos de interés. Estos son los objetivos principales de esta investigación.

\section{Metodología y muestra}

El estudio ha consistido en la selección de un grupo de expertos a quienes se ha preguntado su opinión, anónima, sobre las principales líneas de trabajo e innovación que están apareciendo en la gestión de marcas y en procesos de comunicación asociados a ellas. La metodología utilizada ha sido la realización de un estudio Delphi.

Según Luna et al. (2006: 94), es una técnica para realizar estudios de predicción, fundamentada en opiniones de expertos sobre un tema. Se ha elegido este método porque es el adecuado para el tema analizado, ya que las técnicas cualitativas, se consideran unas de las más completas para recolectar datos, tales como descripciones, observaciones y diálogos sobre cuestiones abiertas.

Como explican Cabero e Infante (2014: 3), los tipos de estudios que se adaptan a la utilización de este método son aquellos que reúnen algunas de las siguientes características: no disponer de información suficiente, que existan problemas de tiempo de los participantes, que se mantenga una cierta heterogeneidad de los participantes y así asegurar la validez de los resultados, y porque los expertos están físicamente dispersos.

También se ha seleccionado porque cumple tres requisitos importantes para la investigación (Taylor \& Meinhardt, 1985; Rowe \&Wright, 1999: 354): 
1. Anonimato: ningún experto conoce la identidad de los otros componentes del panel.

2. Iteración y realimentación controlada: la iteración se consigue al presentar varias veces el mismo cuestionario.

3. Respuesta del grupo en forma estadística: la información que se presenta muestra todas las opiniones de los expertos indicando el grado de acuerdo que se ha obtenido.

Por lo que se puede justificar que el método Delphi es adecuado para abordar el campo de investigación analizado porque 1) es un tema en continua evolución, 2) detecta factores claves para conocer las últimas tendencias relacionadas con las marcas, y 3) los expertos facilitan información de primera mano. De hecho, Rowe y Wright (1999: 363) sostienen que el método Delphi “... es muy adecuado para aquellos problemas en donde la mejor información disponible es la opinión de los expertos".

Una vez planteado el problema y definidos los objetivos que se han descrito en epígrafes precedentes, se seleccionaron a los expertos en función de cuatro temáticas diferentes pero complementarias: académicos y expertos en investigación (profesores universitarios y directores de consultoras de marca), expertos del mundo corporativo (directores de comunicación, CEO, directores de reputación, marca y comunicación y directores de RSC), expertos de marketing y la publicidad (directores de planificación estratégica, directores de marketing y directores creativos), y agencias de medios y expertos en planificación de medios (director de servicios, directores de operaciones, responsables de publicidad y maketing, y head of strategy). Estos grupos heterogéneos de participantes conoce las últimas tendencias relacionadas con la gestión de la comunicación de marca corporativa y comercial.

Se han realizado dos cuestionarios (primera oleada y segunda oleada). La primera oleada se llevó a cabo entre el 27 de marzo y el 15 de abril de 2015, periodo de tiempo que se facilitó a los expertos para contestar a las preguntas de la primera encuesta. Los paneles estaban compuestos por seis o siete especialistas en la materia.

El cuestionario que se envió a los expertos está dividido en dos partes. La primera de ellas trata sobre la gestión corporativa de la marca y la segunda sobre la gestión de las marcas comerciales. A continuación, se puede observar el guión básico de la investigación.

Tabla 1. Resumen de la estructura y contenidos de la primera y segunda oleada del método Delphi aplicados.

\begin{tabular}{|c|c|}
\hline Contenidos primera oleada & Contenidos segunda oleada \\
\hline \multicolumn{2}{|l|}{ Gestión corporativa de las marcas y relación con intangibles } \\
\hline $\begin{array}{l}\text { Importancia de los intangibles. } \\
\text { Comunicación y contenidos corporativos (actuales y potenciales). } \\
\text { Escenarios y plataformas de comunicación } \\
\text { Influencia de los stakeholders. }\end{array}$ & $\begin{array}{l}\text { Plataformas y temáticas concretas para conversar con los públicos. } \\
\text { Jeraquización de públicos en función de la influencia en el diálogo. } \\
\text { Criterios para detectar líderes de opinión. } \\
\text { Estrategias para la recomendación o prescripción. }\end{array}$ \\
\hline \multicolumn{2}{|c|}{ Gestión comercial de las marcas y relación con comunicación y consumidores } \\
\hline $\begin{array}{l}\text { Tendencias de comunicación en relación marca comercial- } \\
\text { consumidor. } \\
\text { Efectos de la publicidad en la marca comercial. } \\
\text { Contenidos y formatos en la comunicación de marca comercial. } \\
\text { Influencia de los consumidores en la gestión y creación de mensajes. }\end{array}$ & $\begin{array}{l}\text { Plataformas y temáticas concretas para conversar con los públicos. } \\
\text { Criterios para detectar líderes de opinión. } \\
\text { Estrategias para la recomendación o prescripción. }\end{array}$ \\
\hline
\end{tabular}

Fuente: Elaboración propia. 
Una vez finalizado el periodo de tiempo para responder al cuestionario, se analizaron las respuestas y qué personas habían contestado a la primera oleada del estudio. Algunos de los errores de diseño fueron los siguientes: se detectaron problemas para responder a algunas de las preguntas planteadas y otras cuestiones eran demasiado amplias. Todo ello sirvió para realizar y mejorar el segundo cuestionario.

Se solicitó participación a 26 expertos. Finalmente, el porcentaje de respuesta a la primera oleada en cada una de las temáticas fue alto (65\% de participación), excepto en el panel de expertos del mundo corporativo, como se observa a continuación:

Tabla 2. Paneles de la primera oleada Delphi de la investigación.

\begin{tabular}{l} 
PANEL 1: Académicos y expertos en investigación (5 expertos) \\
PANEL 2: Expertos del mundo corporativo (2 expertos) \\
PANEL 3: Expertos del mundo del marketing y de la publicidad (5 expertos) \\
\hline PANEL 4: Agencias de medios y expertos en planificación de medios (5 expertos)
\end{tabular}

Fuente: Elaboración propia.

La segunda oleada del estudio se realizó del 5 al 21 de mayo de 2015. El segundo cuestionario que se envió a los expertos se realizó en función de las respuestas obtenidas en la primera oleada y el análisis de las mismas. Este segundo cuestionario consta de seis preguntas diferenciadas entre marca corporativa y marca comercial, y además recoge los matices y particularidades de la primera oleada (Ver Tabla 1).

Los expertos que contestaron a este segundo cuestionario en cada una de los paneles fueron los siguientes:

Tabla 3. Paneles de la segunda oleada Delphi de la investigación.

\begin{tabular}{|l|}
\hline PANEL 1: Académicos y expertos en investigación (5 expertos) \\
\hline PANEL 2: Expertos del mundo corporativo (1 experto) \\
\hline PANEL 3: Expertos del mundo del marketing y de la publicidad (3 expertos) \\
\hline PANEL 4: Agencias de medios y expertos en planificación de medios (4 expertos) \\
\hline
\end{tabular}

Fuente: Elaboración propia.

Una vez realizado el segundo cuestionario se recogieron las respuestas de los expertos y se analizaron.

\section{Resultados}

Los resultados que se presentan a continuación están divididos de acuerdo a los objetivos mencionados, y en función de las respuestas obtenidas por los expertos seleccionados en el Delphi. Se han omitido los nombres y cargos de los especialistas para conservar su anonimato. En primer lugar, se identificarán cuáles son los contenidos y principales plataformas de comunicación de la marca corporativa (3.1) y de la marca comercial (3.2). Posteriormente, se explicarán cómo se entienden 
las relaciones de las empresas-marcas con los consumidores en la actualidad (3.3). Estos puntos se corresponden con los objetivos planteados en la presente investigación y con la información facilitada por los expertos.

\subsection{La marca corporativa: contenido y plataformas}

El marco teórico ha subrayado que la identidad, las acciones de los departamentos de intangibles y las relaciones con los grupos de interés son los principales drivers para la generación de contenido de comunicación corporativa. A la vez, se ha constatado que la literatura mantiene a la publicidad corporativa y a las relaciones públicas como las plataformas clásicas de comunicación corporativa. Este punto describe lo que están haciendo actualmente las empresas y si existen estrategias diferentes a las estrategias descritas en la revisión de la literatura.

En primer lugar, se destacan cuáles son los contenidos transmitidos en la comunicación de la marca corporativa. Los académicos y expertos en investigación sostienen que la marca corporativa es la síntesis del propósito empresarial. Es decir, convierte en conductas valores corporativos. Así, la empresa debe prestar atención a los stakeholders mediante diálogo y escucha, puesto que serán fundamentales para fortalecer la propia identidad y el posterior contenido de la comunicación. Ejemplifican este proceso mediante la presencia empresarial en redes sociales, ya que se trata de espacios donde identidad y modelo de negocio deben alinearse. En consecuencia, aseguran que los contenidos que transmitirá la marca corporativa están profundamente vinculados con la identidad y ésta, a su vez, con los insights recibidos del diálogo y la escucha previa.

En esta misma línea se sitúa el grupo de expertos corporativos, puesto que sostiene que la marca corporativa recibe apoyo de la gestión del resto de intangibles (RSC, cultura corporativa, comunicación corporativa, reputación corporativa), que dan forma a la identidad, y son trasladados al resto de grupos de interés. Este tipo de gestión de contenidos busca que los stakeholders no sólo recomienden los productos de la empresa, sino a toda la organización. Es decir, la comunicación corporativa también busca generar contenidos que favorezcan el word of mouth. Para este grupo de expertos corporativos los principales embajadores de la marca corporativa son los consumidores y empleados y destacan, a diferencia de los otros, la importancia de los contenidos financieros en la comunicación de marcas corporativas.

El grupo de publicidad y marketing asegura que los intangibles aportan un vínculo emocional a la marca corporativa. Aseguran que esta relación puede llegar a superar los beneficios percibidos del producto y mejorar la imagen y reputación. Sin embargo, para conseguirlo se requiere una gran integración en el desempeño de los intangibles con la identidad. Se ejemplifica con la RSC, que solo será bien comunicada cuando esté integrada en la identidad de la empresa. En cualquier caso, sostienen que los valores de la empresa no son protagonistas de los contenidos de la comunicación y que el "hacer" comunica más que el "decir", aunque esta perspectiva no siempre sea asumida por la dirección. Además, alertan de que en ocasiones, la comunicación corporativa es "demasiado protocolaria”. Es decir, que se sustenta en mensajes y formatos que no despiertan emociones o no transmiten adecuadamente los valores de la empresa.

El grupo de agencias de medios y planificación afirma que la estrategia de comunicación de la marca corporativa en relación con los consumidores está muy centrada en vender o conseguir seguidores y engagement.

Esta última es una cuestión que merece la pena subrayar, puesto que, como se ha visto, la marca corporativa está muy vinculada con propósitos, comportamientos y valores. El hecho de emitir un mensaje comercial como parte de la comu- 
nicación corporativa contrasta con los mensajes de generación de confianza y reputación que se emiten a otros grupos de interés, como son: accionistas, empleados, proveedores o sociedad. Si bien es cierto que la fortaleza financiera (vinculada con las ventas) puede generar confianza en públicos como los accionistas, también podría provocar rechazo en colectivos más afines a los valores sostenibles de las marcas corporativas (ONG’s, sociedad en general, comunidades locales).

Tabla 4. Contenidos y plataformas de la marca corporativa.

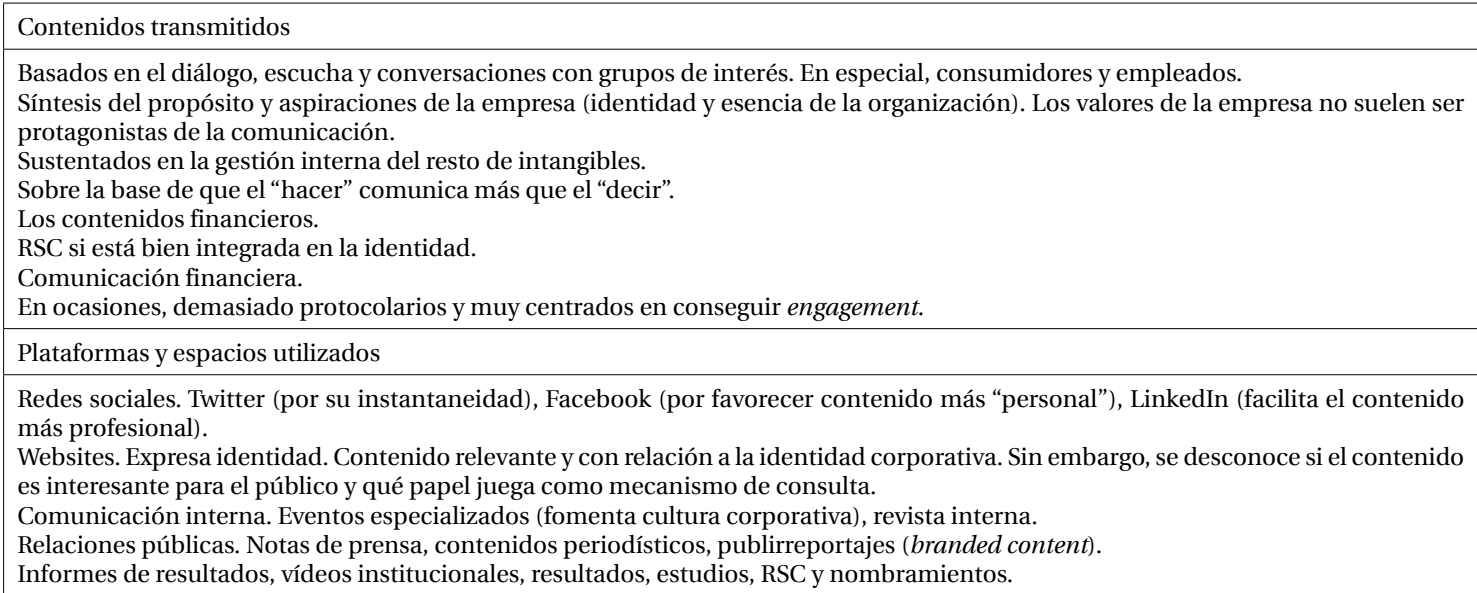

Fuente: Elaboración propia.

En consecuencia, los resultados están alineados con lo analizado en el marco teórico. La marca corporativa surge de la identidad de la empresa y de los comportamientos empresariales. Las acciones del resto de intangibles posibilitan la creación de emociones hacia la marca y la empresa debe cuidar y mantener un diálogo y escucha hacia los grupos de interés. No obstante, se alerta sobre un contenido comunicacional poco emocional y basado, en ocasiones, en atributos financieros.

En segundo lugar, se subrayan las plataformas utilizadas para la comunicación de los contenidos de la marca corporativa. Como ya se ha comentado, una novedad respecto a la revisión de la literatura es la necesidad de generar un contenido que procure el word of mouth, es decir, que los comportamientos generen la recomendación de otros.

Para alcanzar esto, los académicos y expertos en investigación aseguran que las plataformas y temáticas que son utilizadas para llegar a los públicos son: Twitter (instantaneidad), Facebook (difusión de contenido más personal) y LinkedIn (medio de ámbito profesional). Además, se insiste en la pertinencia del website (para expresar identidad y albergar contenido relevante y diferenciador), los eventos especializados (cultura corporativa entre los empleados), la revista interna o el contacto con líderes de opinión a través de experiencias de marca. El grupo de expertos corporativos mantiene ciertas similitudes con el ámbito investigador, puesto que sostiene que las redes sociales ocupan un lugar preponderante en la comunicación de las marcas. No obstante, también inciden en la necesidad de la comunicación interna. 
El grupo de publicidad y marketing asegura que los contenidos explicados se trasladan a los públicos mediante plataformas tradicionales y mundo online (Ej.: Twitter, Linkedin y Slideshare). Tienden a comunicaciones periodísticas en medios impresos, publirreportajes en publicaciones (branded content) sectoriales o noticias generalistas. En la web, la marca corporativa desarrolla todo su contenido e identidad (explicando los valores, proporcionando informes, procurando la transparencia). Sin embargo, los expertos dudan del interés que esta información despierta en el conjunto de los stakeholders y se preguntan si este contenido es visitado asiduamente por las audiencias. Es decir, dudan de que esta transparencia ofrecida a través de la web atraiga la atención.

El grupo de agencias de medios y planificación afirma que las plataformas que utilizan las marcas dependen del sector y del medio (por ejemplo, sostienen que las redes sociales influyen con mensajes más cortos y "tienden a comunicarse banalidades"). Asimismo, afirman que las plataformas tradicionales son habituales en la comunicación corporativa: informes de resultados, vídeos institucionales, resultados de cuentas y estudios, RSC, cambios en cúpula directiva, etc. El problema es que estas plataformas se utilizan de manera unidireccional, "comunican lo que hacen, pero no consideran la integración de su público en sus comunicaciones". Este grupo subraya las siguientes plataformas online para la comunicación corporativa: Facebook, LinkedIn, Twitter, Blog o YouTube.

En términos generales, el análisis de resultados de las tendencias en herramientas y plataformas de comunicación de marca corporativa ha permitido corroborar la importancia de la identidad y de los intangibles en la estrategia de comunicación corporativa. Tal y como se ha descrito, el componente emocional de la marca corporativa viene determinado por la actuación de los intangibles, que, a su vez, deben estar alineados con los valores y objetivos de la marca. En la transmisión de los contenidos, cobran relevancia las redes sociales, aunque la forma de emitir estos mensajes no supone una ruptura, en términos formales, con la comunicación tradicional analizada en el marco teórico. Según Ros (2009), los dos factores más importantes para el gerente de comunicación institucional en el escenario digital son: la satisfacción y la reputación que el entorno de Internet logra gracias a la integración del usuario con la organización.

Es decir, existen nuevas plataformas pero el mensaje sigue siendo emitido exclusivamente a través de la empresa sobre la base de la actuación de los intangibles. Las nuevas tendencias en comunicación buscan los principios de participación y conversación, aunque no se especifica cómo, y sostienen la importancia de apoyarse en el branded content para comunicar mediante la aportación de valor. Simultáneamente, sostienen que las plataformas clásicas, tales como documentos, informes y vídeos corporativos siguen siendo efectivos pero son transmitidos de manera muy unidireccional.

La información recogida permite observar la actual situación de tránsito entre la comunicación tradicional y la que posibilitan los nuevos medios. Asimismo, permite corroborar que la importancia de la identidad es crucial para el desempeño de la gestión interna. No obstante, llama la atención que las tendencias en el ámbito empresarial apenas disten de lo aseverado por la academia en los diversos artículos recogidos en el marca teórico. Por lo tanto, parece necesario profundizar en los contenidos y plataformas de las marcas comerciales y detectar si en este ámbito sí que existen novedades o tendencias determinantes en el panorama de la gestión de marca y comunicación comercial. 


\subsection{La marca comercial: contenido y plataformas}

En primer lugar, se reflejan cuáles son los contenidos transmitidos en la comunicación de la marca comercial. Los académicos y expertos en investigación, sostienen que la comunicación con el consumidor se basa en el denominado "Advertiment”; es decir, asumir que no se puede acudir al público desde un mensaje unidireccional, sino que es necesario conseguir su consentimiento a través de una difusión que implique experiencias positivas, concretamente en punto de venta y en el resto de puntos de contacto de la marca con los clientes. En la utilización de redes sociales hay que realizar un seguimiento de las opiniones que aparecen sobre las marcas en la red, así como las descripciones de experiencias que leen otros usuarios. Los medios tradicionales siguen siendo válidos, siempre y cuando puedan cumplir con los objetivos de negocio. Por otro lado, el grupo de expertos corporativos manifiesta que la marca comercial se centra en la comunicación de beneficios en soportes online y offline. Esto es así porque las redes sociales son altavoces donde la influencia crece de manera eficaz, y las marcas deben gestionarlas de manera adecuada para lograr confianza y credibilidad.

El grupo de expertos provenientes del área de publicidad y marketing sostiene, mayoritariamente, que los principales contenidos que comunican las marcas comerciales son muy diferentes y dependen del sector y de lo que se quiera comunicar. La mayor parte de ellos están relacionados con valores, satisfacción, posicionamiento, imagen, estilos de vida del público al que va dirigido, etc. Los expertos coinciden en que los formatos más utilizados han dejado de ser los tradicionales (aunque se siguen utilizando) para dar paso a otras formas de comunicación como el "branded content", donde la marca crea un contenido para transmitir sus valores y generar comunidad.

Finalmente, el grupo de expertos de agencias de medios y planificación asegura, en conjunto, que la comunicación publicitaria tiene un efecto importante en el valor de la marca comercial. Cuando utiliza el mensaje y los canales correctos es capaz de conseguir un mayor atractivo de marca, incrementar la prescripción y la intención de compra. Afirman que la comunicación publicitaria también contribuye al posicionamiento de marca. Para ser eficaz, la comunicación publicitaria debe combinar estrategias on y off line y debería tener el efecto que la marca haya establecido como objetivo. Junto a formatos más convencionales como el spot, van ganando protagonismo otras formas de comunicación más centradas en contenidos (lanzamiento de productos, promociones, colaboraciones con celebrities, etc.) y los formatos digitales (vídeo online, email marketing, microsites de producto).

En segundo lugar se reflejan las plataformas utilizadas para la comunicación de los contenidos de la marca comercial. Según los académicos y expertos en investigación, las plataformas y temáticas que son más utilizadas para aproximarse a los consumidores son el punto de venta físico (o virtual), que tiene que representar convenientemente la proposición de la marca. También destacan los entornos online y los medios tradicionales. No reflejan una plataforma y temática concreta, sino que resaltan la importancia de centrarse en estrategias ad hoc que creen comunidad.

Sin embargo, el grupo de expertos corporativos acentúa la importancia de las redes sociales para llegar al consumidor, y también manifiesta la necesidad de incluir un servicio de atención al cliente multiplataforma, y la incorporación de personajes conocidos para incrementar la notoriedad y credibilidad de la marca. 
Tanto el grupo del área de publicidad y marketing como el de las agencias de medios y planificación, afirman que las principales plataformas que debe emplear una marca comercial para conversar con sus públicos se centran en el mundo online. Entre las citadas destacan Facebook, Instagram, Tumblr o Twitter. El grupo de expertos de publicidad y marketing también señalan algunas plataformas vinculadas con los grandes medios y las Relaciones Públicas. Y el grupo de medios y planificación hace hincapié en que es muy importante que la marca no vaya a redes sociales generalistas sin un análisis previo de dónde se encuentra su público potencial "que haya usuarios en redes sociales no quiere decir que haya clientes". Deben abordar las temáticas que han identificado en investigación que interesan o demandan sus públicos.

Tabla 5. Contenidos y plataformas de la marca comercial.

\begin{tabular}{|l|}
\hline Contenidos transmitidos \\
\hline Beneficios y prestaciones asociados al producto. \\
Los delimitados por el sector. \\
Valores de la marca. \\
Posicionamiento e imagen de la marca. \\
Precio. \\
Estilos de vida del público objetivo. \\
Advertiment: no se puede llegar desde la unidireccionalidad, sino mediante experiencias positivas que consigan el "consenti- \\
miento" del impacto recibido. \\
\hline Plataformas utilizadas \\
\hline Punto de venta físico que refleje proposición de marca. \\
Punto de venta online. Además de proposición de marca, debe proporcionar seguridad, disponibilidad y ofertas. \\
Entornos online. Control de opiniones, comentarios y experiencias de marca que aparecen en la Red. Objetivo: crear confianza \\
y credibilidad. \\
Formatos digitales: video online, email marketing, microsites de producto, sorteos, concursos en redes sociales. \\
Redes sociales. Destacan Facebook, Instagram, Tumbl o Twitter. Alerta sobre análisis previo para detectar público potencial y no \\
tener presencia sin fundamento. \\
Branded content. Creación de contenido para transmitir valores y crear comunidad. \\
Publicidad tradicional (spot). Da valor de marca, atractivo e incrementa la prescripción e intención de compra. \\
Lanzamiento de productos. \\
Promociones. \\
Colaboraciones con celebrities. \\
Trucos y consejos con productos y servicios.
\end{tabular}

Fuente: Elaboración propia.

Los resultados muestran similitud con los autores citados en el marco teórico, al comprobar que los contenidos que comunican las marcas de producto están vinculados con la identidad corporativa. Además, es importante potenciar una proposición de venta que se ampare en atributos objetivos del producto, pero también en comportamientos. Todo este proceso de cambio en la forma de comunicar los productos viene determinado por la influencia que han ejercido los públicos en la gestión de marca comercial. Ahora la gestión tiene que basarse en la co-creación y en el conocimiento obtenido a través de las redes sociales. Aunque las redes sociales ocupan un lugar preponderante en la comunicación de las marcas, cabe destacar la comunicación interna y la comunicación financiera como otras estrategias de comunicación para llegar a los diferentes stakeholders. Y es importante señalar que los principales públicos que más influyen en el diálogo creado por las 
marcas en el ámbito digital son los consumidores y empleados, a considerar por las marcas en su estrategia de comunicación. En el caso de las marcas modernas, los consumidores no sólo completan el mensaje de marca, sino que son parte fundamental, sin ellos no hay mensajes. Por eso los consumidores son los públicos que más influyen en el diálogo iniciado por las marcas.

La estrategia de comunicación de la marca en relación con los consumidores está muy centrada en vender o en conseguir seguidores y engagement (no se destacan contenidos corporativos específicos). Por eso debe potenciarse el uso de nuevos canales y formatos. Los consumidores esperan que las marcas les escuchen e incorporen toda esta información para mejorar la relación y establecer fórmulas que les sorprendan en los nuevos productos y servicios que lanzan. Lo difícil es interpretar toda esta información y sistematizarla para que revierta en unas estrategias más eficaces.

Por eso, hay que dar importancia a las estrategias de comunicación, diseñar los pasos a seguir, y utilizar diferentes estrategias en el mercado para llegar a sus públicos, tal y como se refleja en el marco teórico. De esa forma, la comunicación publicitaria tendrá un efecto importante en el valor de la marca comercial, cuando utilice el mensaje y los canales correctos, porque será capaz de conseguir un mayor atractivo de marca, y también contribuirá al posicionamiento de marca.

Tras describir los resultados relativos al bloque de preguntas concerniente a los objetivos vinculados con las tendencias en contenidos y plataformas de comunicación de marcas corporativa y comercial, se procede a explicar cuáles son las tendencias en la relación con los consumidores, puesto que es un grupo de interés relevante para las marcas.

\subsection{Tendencias en la relación con los consumidores. El 'diálogo'.}

Aunque el marco teórico ha demostrado que la marca corporativa tiene un enfoque multi- stakeholder, la elección de un público como el de los consumidores se debe a que tanto marca comercial como corporativa comparten la intención de investigar las expectativas de este tipo de audiencia y el deseo de saber comunicar sus acciones. Atender las formas de diálogo con otros grupos de interés pondría el foco de atención en las marcas corporativas. En esta investigación se observan los terrenos compartidos entre ambas. Tal y como se ha dicho, en arquitecturas de marca branded house y endorse brand, la marca corporativa también guarda relación con las percepciones que despiertan la comunicación de productos o servicios, puesto que la identidad corporativa respalda en menor o mayor grado la comunicación de las marcas comerciales.

En la relación marca-consumidor, la mayoría de grupos sostiene que es necesario comunicar mediante el diálogo. Aunque la conversación tiene que tener en cuenta a todos los stakeholders, los grupos analizados aseguran que las grandes marcas escuchan la opinión del consumidor al crear mensajes y realizan investigaciones para identificar sus preferencias.

Sin embargo, destacan que es muy difícil interpretar esta información y sistematizarla para que revierta en estrategias eficaces. Se afirma que los consumidores esperan que las marcas escuchen e incorporen la información para mejorar la relación y establecer fórmulas que sorprendan, tanto en productos como en servicios ofertados. Además, las marcas deben atender a los nuevos prescriptores sociales: bloggers, instagramers o youtubers. Sus opiniones pueden ser determinantes para la promoción de productos y servicios. 
Según los datos obtenidos de los expertos de los diversos ámbitos, parece adecuado sostener que el denominado diálogo domina las relaciones de las marcas con los consumidores. A continuación se especifica cómo se materializa esa relación en sus diferentes espacios o estrategias.

\subsubsection{La jerarquización del diálogo. Detección de líderes de opinión}

Una gran parte de los expertos consultados asegura que las marcas tienen que detectar a los líderes de opinión. En especial, en los nuevos medios. Los expertos corporativos subrayan que la influencia de los líderes de opinión en este medio se produce así: los usuarios son tratados como iguales. Estos iguales generan recomendación directa y confianza en las marcas. Los influenciadores (influencers), muchas veces empleados y clientes, llegan a los no clientes y se extiende después a toda la sociedad. Esto es así porque, según los expertos consultados, el público genera más contenido que la propia marca. El grupo de expertos en publicidad incide en que los públicos siempre han influido en la gestión de las marcas, pero actualmente más porque pueden expresar públicamente su opinión. Para detectar a los líderes se tiene una perspectiva muy centrada en redes sociales.

En este sentido, los académicos e investigadores subrayan que hay que monitorizar a personas que en redes sociales tengan abundantes seguidores pero que, además, generen diálogo, participen en el debate sociopolítico y cultural; y que sus artículos y tweets sean leídos y consigan "me gusta" o retweets. Es decir, que generen conversaciones y menciones. Cualitativamente, también se destaca el análisis de la calidad de la interacción con los públicos, así como su perfil profesional y actuación pública. En esta misma línea, el grupo de publicidad y marketing asegura que el criterio nunca es su número de followers, sino su capacidad para transmitir credibilidad en un mensaje.

En definitiva, no llega a existir total consenso entre los grupos, puesto que algunos prefieren los criterios cuantitativos y otros los cualitativos. Sin embargo, sí que se observa una clara tendencia a comunicar a través de las recomendaciones o apoyos que se obtienen mediante los influencers. El problema es que no llega a quedar claro cómo localizarlos. En lo que sí parece haber más consenso es en la forma de acercarse a ellos. A continuación, se detalla.

\subsubsection{El papel de la empresa: contar qué se hace y por qué se hace}

Los expertos en publicidad y marketing dicen que se debe potenciar una proposición de venta que se ampare en atributos objetivos del producto, pero también en comportamientos. Además, los expertos corporativos aseguran que la diferenciación se encuentra en la gestión de la marca corporativa, ya que permite construir personalidad única mediante valores que deben ser comunicados. Es necesario para las marcas generar un modelo de influencia que construya "creencias compartidas con todos sus públicos". De este modo se integran marca comercial y marca corporativa en la comunicación.

En esencia, lo que los grupos de expertos consultados sostienen es que, más allá del canal o el medio, las marcas deben ser más honestas y transparentes, emplear territorios, valores y temáticas que interesen al público y que consigan su implicación. Por lo tanto, una identidad honesta es clave para poder aproximarse a la recomendación del consumidor. 


\subsubsection{El desarrollo del diálogo. Las plataformas online y las experiencias de calidad}

La forma en que la organización está presente en Internet implica compromiso por parte de los responsables de la organización. Según los expertos de agencias de medios y de planificación, el mundo digital conlleva un abanico de canales que hacen más compleja la estrategia publicitaria de marca. Esta debe atender a lo que dicen, a cómo contestan y reaccionan sus públicos. Las marcas deben demostrar capacidad para estar cerca de sus clientes, escucharles y detectar necesidades $\mathrm{y}$ formas de vivir y pensar.

Todos los grupos alertan de que estas estrategias requieren formación y conocimiento sobre las temáticas que interesan a los públicos. Según menciona el grupo de expertos en planificación de medios, no se trata sólo de generar contenidos mecánicamente, sino de integrar estrategias globales de comunicación a nivel digital, consiguiendo generar contenidos relevantes capaces de captar la atención de un consumidor cada vez más saturado por la publicidad.

Los expertos corporativos recomiendan no solo redes sociales para llegar al consumidor, sino el servicio de atención al cliente multiplataforma, como manera de transmitir contenidos y experiencias de calidad. La mayor parte de los expertos del área de publicidad y marketing resaltan que las nuevas tendencias en la relación marca-producto-consumidor están vinculadas con Internet, pero también en aportar contenido y experiencia de marca con calidad. Se destaca el "Storydoing" como técnica para compartir una historia con acciones, no con conversaciones. Según los expertos en publicidad y marketing, el lugar que una marca quiere ocupar en la mente del consumidor ya no lo define la marca, sino que viene dado por su aportación a la vida de las personas.

Por último, los expertos en investigación y académicos sostienen que es válida la técnica de envío de prototipos, la personalización de productos, y la invitación a eventos para disfrutar de la experiencia de marca. También surgen estrategias como transmedia, storytelling, etc. que proponen contenido relevante que favorece la prescripción y ofrecen experiencias y herramientas útiles, como el branded content, geolocalización, recomendaciones personalizadas, etc. Asimismo, se concretan acciones como el desarrollo de sorteos y concursos, vídeos virales, aparecer en blogs de influencers y en sus perfiles de redes sociales, etc.

En consecuencia, parece necesario que el diálogo se centre en localizar a los líderes, demostrar honestidad y procurar todo un universo de significado transversal, basado en comunicación, experiencias y mensajes transmedia que se apoyen en la identidad o esencia de la organización.

\subsubsection{Las consecuencias del diálogo. Co-creación de marcas}

Todo ese proceso previo de gestión de relaciones se basa en los principios de co-creación e incorporación del conocimiento que dan las redes sociales. El diálogo, por tanto, debe basarse en los principios de co-creación de marca y la personalización de productos, servicios o la forma de comunicarse con cada cliente. Se trata de segmentar más y adaptar el mensaje mejor, sin olvidar la originalidad. Es decir, la información que circula ya no es sólo de la compañía, sino de lo que opinan los públicos y los contenidos que generan. Los expertos coinciden en destacar que los consumidores no sólo completan el mensaje de marca, sino que son parte fundamental de la misma. Sin ellos no hay mensajes. Por esta razón, cobran importancia las acciones previamente mencionadas (transmedia, storytelling, branded content, geolocalización, personaliza- 
ción...) ya que las marcas generan contenido relevante que favorece la prescripción y ofrece experiencias y herramientas útiles.

Es subrayable que gran parte de los resultados obtenidos guardan estrecha relación con la comunicación en el ámbito online, y bajo una perspectiva muy vinculada con las redes sociales. Cabe mencionar, aunque no haya sido muy prolífico en los resultados, dónde quedan las perspectivas de la comunicación publicitaria tradicional. Así, los expertos afirman que el peso de la comunicación pagada sobre el valor de la marca es cada vez menor y, dependiendo de la reputación, la publicidad puede llegar a ser negativa. No obstante, como curiosamente menciona el grupo de expertos en el ámbito corporativo, las tendencias de comunicación de producto se basan en el cortoplacismo y en la integración de medios para lograr un impacto inmediato, por lo que la publicidad es eficaz en este aspecto para motivar la compra.

\section{Conclusiones y futuras investigaciones}

Los contenidos que transmiten las marcas corporativas tienen su origen en la identidad corporativa y las acciones derivadas de la gestión de intangibles. Por lo que las tendencias en contenidos siguen muy alineadas con los postulados que durante años ha mantenido la investigación académica y que se ha detallado en el marco teórico. El llamado diálogo y escucha con los grupos de interés se presenta como fundamental.

Sin embargo, a la hora de definir la metodología de la conversación, los expertos consultados no son capaces de detallar el fenómeno ni estipular los criterios con los que detectar a los líderes de opinión de las redes sociales (cualitativo-cuantitativo). Por lo que la situación actual presenta una oportunidad para la investigación, puesto que se conoce la importancia de escuchar pero no hay metodologías claras para incorporar los insights (conectar la marca con el consumidor) en la gestión de la marca.

Por tanto, los resultados permiten concluir que las empresas han incorporado en su discurso la teoría de los grupos de interés pero que aún no han sabido canalizar el ámbito teórico con la dimensión práctica. Esto es así, porque a pesar de desarrollar espacios adecuados para el diálogo, las empresas conversan sobre temas que interesan únicamente a la organización. Por lo que no se favorece un diálogo abierto en el que los grupos de interés puedan expresar claramente sus posturas (Illia et al, 2015). Asimismo, las empresas tampoco presentan un verdadero análisis de las pretensiones de los grupos de interés según los postulados de los artículos fundamentales (Mitchell, Agle \& Wood, 1997), sino que buscan la recomendación de influencers de manera directa, dejando de lado gran parte de las opiniones del resto de consumidores. Por lo tanto, se quiere dialogar pero la forma de realizarlo es mediante una comunicación unidireccional que favorece los "me gusta" o "retweets" pero que no habilita un verdadero espacio en el que compartir inquietudes.

Para conseguir esta adhesión a la marca, se apuesta por ser fiel a un estilo comunicativo que aporte valor. En concreto, la elaboración de branded content. Los expertos, mayoritariamente, apuestan por una comunicación de marca corporativa que ofrezca un contenido de interés para los públicos. En este caso, a través de la generación de espacios de interés que no sean meramente comerciales. En cualquier caso, el uso de redes sociales de utilización masiva, las relaciones públicas, la comunicación interna o la comunicación financiera siguen siendo habituales en la gestión de marca. No obstante, se subraya que la website es un espacio idóneo para transmitir la identidad corporativa. Sin embargo, los expertos ponen en 
duda el interés que suscita la información corporativa albergada en la website. Es decir, desconfían del atractivo que puede tener para los grupos de interés.

En resumen, la comunicación de marca corporativa está muy vinculada con la actuación de los intangibles. Los expertos afirman que los contenidos de las marcas deben tener un propósito y aspiraciones claras. Para ello, se deberá prestar especial atención al desarrollo y fortalecimiento de la identidad. Esta esencia de la marca corporativa se nutre de las acciones que realizan el resto de intangibles de la empresa. En otras palabras, son los intangibles los que están en posición de generar un contenido que posteriormente será comunicado. Así, las empresas hacen y, posteriormente, comunican. En todo este conjunto de relaciones, los contenidos relacionados con el ámbito financiero siguen teniendo mucha fuerza en la gestión de la comunicación de las marcas comerciales.

Los resultados hallados permiten sostener que las empresas han incorporado en su gestión de marca corporativa una serie de postulados, basados en la importancia de la identidad, que están alineados con lo que los investigadores vienen desarrollando en los últimos años. Por esta razón, se ve una asociación clara entre el marco teórico y lo que las marcas corporativas están realizando a nivel de contenidos. Sin embargo, no se observa que la práctica empresarial vaya por delante de las investigaciones académicas.

Finalmente, las plataformas utilizadas son las encargadas de transmitir este contenido corporativo. Sin embargo, además de la utilización de redes sociales generalistas y la intención de llegar a los influencers, no parece que los usos de los nuevos medios sean muy diferentes a lo que se venía realizando con la publicidad tradicional. Es decir, se trata de mensajes unidireccionales apoyados en plataformas que dicen ser bidireccionales.

Por otro lado, los contenidos transmitidos por las marcas comerciales para entablar conversaciones con el consumidor se basan en el denominado "Advertiment", es necesario crear experiencias positivas en los puntos de contacto con los clientes, sobre todo, en el punto de venta.

La tendencia de comunicación de producto se basa en el cortoplacismo y en la integración de medios para lograr un impacto inmediato, por lo que la publicidad es eficiente en este aspecto para motivar la compra.

También es importante resaltar que las nuevas tendencias en la relación marca-producto-consumidor están vinculadas con el mundo digital. Se destaca el storydoing como una técnica para compartir una historia con acciones, no con conversaciones. El lugar que una marca quiere ocupar en la mente del consumidor ya no lo define la marca, sino que viene dado en función del valor que aporte en la vida de las personas. Se concluye diciendo que para conseguir la recomendación de sus públicos, las marcas deben ser más honestas y transparentes.

Las principales plataformas que emplea la marca comercial para conversar con sus públicos están relacionadas en su mayor parte con el mundo online. Pero también hay algunas vinculadas con los grandes medios, las Relaciones Públicas y el punto de venta físico. En la utilización de las redes sociales hay que controlar las opiniones que aparecen sobre las marcas en la red, así como las descripciones de experiencia que leen otros usuarios, porque se han convertido en el espacio donde los ciudadanos se manifiestan como defensores o detractores de las marcas. 
La principal conclusión, en tendencias en relación con los consumidores, radica en la necesidad de diálogo con los grupos de interés y el amplio número de opciones que esta estrategia tendrá tanto en la marca corporativa como en la comercial. Se observa que la preocupación por el hacer y el decir es una constante en todo lo relativo a la gestión de marcas corporativas.

Uno de los aspectos que cobra especial interés es la dicotomía entre querer dialogar pero no encontrar los modos adecuados de hacerlo. Por lo tanto, la necesidad de definir la forma en la que hablar con los stakeholders es fundamental para el futuro. Se trata de conseguir articular una correcta gestión de la identidad para así poder desarrollar acciones que lleguen a los grupos de interés a través de mensajes que en muchas ocasiones sean viralizados a través de las redes sociales y mediante los llamados influencers o nuevos líderes de opinión. Así, surgen iniciativas relacionadas como el storytelling, el advertiment o el uso de Facebook, Twitter o Instagram para hablar con sus públicos. Uno de los aspectos más relevantes hallados en la investigación es que la detección de los líderes de opinión en redes sociales se encuentra en proceso de construcción. No existe consenso a la hora de determinar qué variables son más importantes para reconocer a una persona con relevancia en redes sociales. Algunos expertos abogan por criterios cualitativos y otros por criterios cuantitativos (followers, me gusta, retweets, etc.).

Más allá de estrategias de comunicación, necesarias para realizar una comunicación eficaz, conseguir ventaja competitiva y diferenciar la marca en el mercado, la mayoría de expertos aboga por sistemas de gestión de marca que tengan en cuenta a los públicos. Esta escucha no sólo se debe traducir en el devenir de la marca, sino en la gestión del resto de intangibles. Ideas como co-creación, diálogo con los grupos de interés y contacto con líderes de opinión prestan atención a la experiencia de marca, con un matiz muy vinculado a la idea de "ser" para "ser reconocido". La información que circula ya no es sólo de la compañía, sino de lo que opinan los públicos y los contenidos que ellos generan.

El estudio aporta una serie de reflexiones y cuestiones importantes a tener en cuenta, sobre la marca corporativa y comercial. Tales como: contenidos y plataformas de comunicación utilizadas por las marcas, y la relación que establecen las empresas con los consumidores. Futuras líneas de investigación deberían considerar algunas de estas ideas para permitir conocer más en profundidad lo que buscan y necesitan los públicos y lo que ofrecen las marcas. Es decir, buscar la conexión entre la marca y el consumidor.

Los resultados facilitan varias líneas de investigación futura para ser analizadas en los nuevos estudios:

- Plataformas y mediciones de diálogo con grupos de interés. Esto es relevante para saber qué plataformas utilizan las marcas y cómo analizan estos datos, teniendo en cuenta la importancia que se da a la conversación para obtener insights.

- Análisis e identificación de líderes de opinión en los nuevos medios. Estos líderes se han convertido en influencers cuyos impactos en las marcas deben ser analizados. Las técnicas cualitativas y cuantitativas proporcionan conocimiento, pero es oportuno medir efectivamente su influencia en los nuevos medios.

- Integración de la experiencia de la marca en nuevos medios y estrategias, tales como: storytellingy advergaming. Se enfatiza la relevancia del storydoing, una estrategia que se usa en las experiencias de marca y su análisis a través de nuevos formatos de comunicación. 
- Modelos de gestión que integran la identidad de marca en entornos online. La investigación destaca la necesidad de involucrar a la alta dirección en la representación de la compañía en las redes sociales y las conversaciones online. Por lo tanto, es necesario saber cómo integrar la realidad del departamento de gestión de marca, más allá del uso de la subcontratación y la responsabilidad del administrador de la comunidad.

\section{Referencias bibliográficas}

Aaker, D. A. (2004). Brand Portfolio Strategy. New York: The Free Press.

Aaker, D.A. \& Joachimsthaler, E. 2000a. Brand Leadership. New York: The Free Press.

Aaker, D.A., \& Joachimsthaler, E. 2000b. The Brand Relationship Spectrum: The Key to Brand Architecture Challenge. California Management Review, 42(4): 8-23

Aaker, D. A. \& Joachimsthaler, E. (2004). Liderazgo de marca. Bilbao: Deusto.

Alard, J., \& Monfort, A. (Coord.) (2017). Plan de comunicación on y offen la práctica. Madrid: ESIC.

Alberto Pérez, R. (2008). Estrategias de comunicación. Barcelona: Ariel

Ansoff, H.I. (1965). Corporate Strategy: An analytic approach to business policy for growth and expansion. New York: McGraw-Hill

Arroyo, I., Martín, R., \& Farfán, J. (2013). Usos, percepciones y potencialidades de las redes sociales en la construcción de las marcas. Doxa Comunicación: revista interdisciplinar de estudios de comunicación y ciencias sociales. XVI. 129-149

Balmer, J.M.T., \& Gray, E. (2003). Corporate Brands: What are they? What of them? European Journal of Marketing, 37, 972-997.

Barnard, C.I. (1938/1968). The Functions of the Executive. Cambridge, MA: Harvard University Press,

Barney, J. B. (1986). Organizational culture: can it be a source of sustained competitive advantage?, Academy of Management Review, 11(3), 656-665.

Cabero, J. \& Infante, A. (2014). Empleo del método Delphi y su empleo en la investigación en comunicación y educación. EDUTEC, Revista Electrónica de Tecnología Educativa, 48. Retrieved from http://edutec.rediris.es/Revelec2/Revelec48/ n48_Cabero_Infante.html

Cable, D. M., \& Graham, M. (2000). The determinants of organizational reputation: a job search perspective. Journal of Organizational Behavior, 21, 929-947.

Capriotti, P. (2007). Planificación del perfil de identidad corporativa para marcas globales. In P. Capriotti (Ed.), Gestión de la marca corporativa. Buenos Aires: La Crujía.

Carreras, E., Alloza, Á., \& Carreras, A. (2013). Corporate Reputation. Madrid: LID.

Chomvilailuk, R., \& Butcher, K. (2018). The impact of strategic CSR marketing communications on customer engagement. Marketing Intelligence \& Planning, 36(7), 764-777. 
Cohen, D. (1986). Trademark Strategy, Journal of Marketing, 50, 61-74

Colleoni, E. CSR communication strategies for organizational legitimacy in social media, (2013). Corporate Communications: An International Journal, Vol. 18 Iss 2, pp. 228-248.

Day, G.S., \&Wensley, R. (1983). Marketing Theory wtih a Strategic Orientation, Journal of Marketing, 47, 79-89

Falkheimer,J. (2014). The power of strategic communication in organizational development. International Journal of Quality and Service Sciences, 6(2/3), 124-133.

Fernández, J.L. \& Bajo, A. (2012). La teoría del stakeholder o de los grupos de interés, pieza clave de la RSE, del éxito empresarial y de la sostenibilidad. Revista Internacional de Investigación en Comunicación aDResearch ESIC, 6(6), 130-143.

Freeman, R.E. (1984). Stratgic Management. A stakeholder approach. Boston: Pitman Ballinger.

Galbreath, J. (2010), Drivers of corporate social responsibility: the role of formal strategic planning and firm culture. British Journal of Management, 21(2), 511-525.

García Uceda. M. (2001). Las claves de la publicidad. Madrid: ESIC

Hallahan, K., Holtzhausen, D., van Ruler, B., Verc`ic``, D. \& Sriramesh, K. (2007). Defining strategic communication, International Journal of Strategic Communication, 1(1), 3-35.

Hatch, M. J. \& Schultz, M. (2003). Bringing the corporation into corporate branding. European Journal of Marketing, 37(7), 1041-1064.

Hatch, M. J. \& Schultz, M. (2010). Esencia de marca. Madrid: Lid.

Highhouse, S., Zickar, M. J., Thorsteinson, T. J., Stierwalk, S. L., \& Slaughter, J. E. (1999). Assessing company employment image: an example in the fast food industry. Personnel Psychology, 52, 151-172.

Hulberg, J. (2006). Integrating corporate branding and sociological paradigms: A literature study. Journal of Brand Management, 14(1), 60-73.

Hurst, B., \& Ihlen, Ø. (2018). Corporate Social Responsibility and Engagement. In K. A. Johnston \& M. Taylor (Eds.), Handbook of communication engagement (pp. 133-152). Malden, MA: Wiley-Blackwell.

Illia, L., Romenti, S., Rodríguez-Cánovas, B., Murtarelli, G., \& Carroll, C. E. (2015). Exploring Corporations' Dialogue About CSR in the Digital Era. Journal of Business Ethics, 1-20.

Ind, N. (1997). The corporate brand. Nueva York: New York University Press.

Knox, S. \& Bickerton, D. (2003). The six conventions of corporate branding. European Journal of Marketing, 37(7), 998-1016.

Kollat, J., \& Farache, F. (2017). Achieving consumer trust on Twitter via CSR communication. Journal of Consumer Marketing, 34(6), 505-514.

Kotler, P., \& Keller, K.L. (2006). Marketing Management (12th ed). New Jersey: Prentice- Hall.

Lindgreen, A., Xu, Y., Maon, F., \& Wilcock, J. (2012). Corporate social responsibility brand leadership: a multiple case study. European journal of marketing, 46(7/8), 965-993. 
López, B. \& Fornés, G. (2015): Corporate social responsibility in emerging markets. Case studies of Spanish MNCs in Latin America. European Business Review, Vol. 27, Issue, 2.

López, B. \& Villagra, N. (2017). Corporate competitiveness based on Sustainability and CSR values: Case studies of Spanish MNCs. Strategic innovative marketing, pp.309-314. Springer.

Luna Huertas, P., Infante Moro A., \& Martínez López, FJ. (2006). Los Delphi como fundamento metodológico predictivo para la investigación en sistemas de información y tecnologías de la información (IS-IT). Pixel-Bit. Revista de Medios y Educación. Sevilla, 26, 89-112. Retrieved from http://redined.mecd.gob.es/xmlui/bitstream/handle/11162/95167/00820123017135. pdf?sequence $=1$

Mitchell, R.K., Agle, B.R. \&Wood, D.J. (1997). Towards a theory of stakeholder identification and salience: defining the principle of who and what really counts. Academy of Management Review, 22(04), 853-886.

Monfort, A., \& Villagra, N. (2016). Corporate social responsability and corporate foundations in building responsible brands. El profesional de la información (EPI), 25(5), 767-777.

Ortega Martínez, E. (1997). La comunicación publicitaria. Madrid: Pirámide.

Osler, R., (2003). Infusing business and brand strategies into account planning. Marketing Intelligence \& Planning, 21(7), 435-439.

Pintado, T.; Sánchez, J. \& Merino, M.J. (2010). Comunicación online. In J. Sánchez Herrera y T. Pintado (Eds.). Nuevas tendencias en comunicación. (pp.42-61). Pozuelo de Alarcón (Madrid): ESIC.

Porter, M.E. (2002). Estrategia competitiva. Técnica para el análisis de los sectores industriales y de la competencia. México: Continental

Reinares, P. \& Calvo, S. (2001). Gestión de la comunicación comercial. Madrid: McGraw-Hill.

Ros Diego, V. J. (2009). Modelo para la gestión de marca en Internet. Doxa Comunicación: revista interdisciplinar de estudios de comunicación y ciencias sociales. IX. 195-203

Rowe, G. y Wright, G. (1999) The Delphi technique as a forescasting tool: issues and analysis. International Journal of Forecasting. 15, 353-375.

http://forecastingprinciples.com/files/delphi\%20technique\%20Rowe\%20Wright.pdf

Schein, Edgar H. (1984). Coming to a New Awareness of Organizational Culture, Sloan Management Review, 25(2), 3-16.

Schultz, M. \& de Chernatony, L. (2002). Introduction to the challenges of corporate branding. Corporate Reputation Review, 5(2), 105.

Sebastián, A., Ayestarán, R., \& Rangel, C. (2012). Planificación estratégica y gestión de la publicidad. Conectando con el consumidor. Madrid: ESIC

Taylor, R.E. \& Meinhardt, D. J. (1985). Definig computer information needs for small business: A delphi method. Journal of Small Business Management, 23, 3-9. 
Tuñez López, M. (2012). La gestión de la comunicación en las organizaciones. Zamora: Comunicación Social Ediciones y Publicaciones

Villagra, N. \& López, B. (2013). Analysis of values and communication of the responsible brands. Corporate brand strategies for sustainability. Communication \& Society, 26(1), 201-226.

Wartick, S.L. \& Wood, D.J. (1998). International Business and Society. Malden: Blackwell.

Weick, K.E. (1979). The Social Psychology of Organizing (2nd ed.), Reading, MA: Addison-Wesley.

Windsor, D. (2006), "Corporate social responsibility: three key approaches", Journal of Management Studies, 43(1), 93-114.

\section{Nota:}

El artículo recoge parte de los resultados de una Investigación financiada y aprobada por el Ministerio de Economía de España, referencia CSO2013-46410-R. Programa Estatal de Investigación, Desarrollo e Innovación. Proyectos de I+D+I 
